\title{
Piezo-spectroscopic induced perturbations for defects in cubic crystals under uniaxial stress applied along arbitrary low-symmetry crystal directions
}

\author{
K G McGuigan $\dagger \|$, E McGlynn $\ddagger$, F O’Cairbre§, J Love $\S$ and M O Henry \\ † Department of Physics, Royal College of Surgeons in Ireland, 123 St Stephens' Green, \\ Dublin 2, Ireland \\ $\doteqdot$ School of Physical Sciences/National Centre for Plasma Science and Technology (NCPST), \\ Dublin City University, Glasnevin, Dublin 9, Ireland \\ $\S$ Department of Mathematics, National University of Ireland, Maynooth, Co. Kildare, Ireland \\ E-mail: kmcguigan@rcsi.ie \\ Received 13 April 2000, in final form 8 June 2000
}

\begin{abstract}
A modification to supplement the experimental procedure required to identify the symmetry of point and axial defects in silicon via photoluminescent piezo-spectroscopy is reported. The modification requires that uniaxial stress be applied along a crystal direction of very low symmetry such that all orientational degeneracy is removed. Theoretical shift rate equations for all orientationally degenerate transitions and electronically degenerate states in a tetrahedral host all orientationally degenerate transitions and electronically degenerate states in a tetrahedral host
crystal are calculated for stress applied along an arbitrary direction $(X Y Z)$. These calculations are corroborated against the predictions of previous work and are used to successfully predict the stress induced splitting of the $983.21 \mathrm{MeV} \mathrm{Cd}_{A}$ line in silicon under stress along the (136) crystal axis. The advantages this low-symmetry-axis perturbation-spectroscopy (LSAPS) technique offers in conjunction with standard uniaxial stress analysis techniques are discussed.
\end{abstract}

\section{Introduction}

Piezo-spectroscopy is a widely used technique for identifying the electronic environment and symmetry properties of optically active defects in crystals [1-3]. The technique involves applying a uniaxial stress along the major axis of a parallelepiped-shaped, oriented crystal sample. The applied stress causes optical transitions that originate at defects within the crystal to shift or split into a number of sub-components. The symmetry of the defect and the electronic nature of the transition can then in principle be determined from the number, shift rate and polarization intensities of the stress-induced components. Piezo-spectroscopic studies of defects in zincblende or diamond-type crystals usually require stress to be applied to three oriented samples aligned along the (1111), (1 110$)$ and $\left(\begin{array}{lll}0 & 0 & 1\end{array}\right)$ crystal axes (though occasionally only two of these axes are used), in order to uniquely identify the observed transition and defect symmetry [3-5].

There are a number of practical difficulties involved in making reliable stress measurements. Uniaxial stress studies of the photoluminescence (PL) or absorption spectra originating from axial defects in many semiconductors are complicated by the necessity to apply high stresses to oriented samples maintained at liquid helium temperatures in order to

\| Corresponding author. 
resolve the spectral detail required for reliable results [6]. Further complications occur if the stress and/or the sample are misaligned either by incorrect mounting within the stress cell or faulty alignment of the sample during the orientation procedure. Consequently, obtaining complete uniaxial stress data for each of the (1 111$),\left(\begin{array}{lll}1 & 1 & 0\end{array}\right)$ and ( 0001$)$ crystal directions can be a lengthy procedure.

Apart from these practical considerations there are a number of more fundamental difficulties associated with deducing the defect symmetry from traditional stress methods only. Firstly, the presence of higher-lying excited states which may interact with the lowest excited state to produce level anti-crossing effects complicates matters to a great extent. The additional free parameters describing the coupling between levels and the behaviour of the higher level(s) (on which data are often quite poor due to thermalization effects at low temperatures) mean that unambiguous identification of the symmetry from the normal procedures is often not possible. An example of such possible ambiguity would be the cases of transitions at centres with monoclinic I symmetry and A-E transitions at trigonal centres, both of which split into two, three and four components under ( $\left.\begin{array}{llll}0 & 0 & 1\end{array}\right),\left(\begin{array}{lll}1 & 1 & 1\end{array}\right)$ and (1 110$)$ stress respectively [3]. In the presence of inter-level repulsion the additional free parameters mean that in many cases these two possibilities may not be unambiguously distinguished [8].

While the (polarized) intensities of the various sub-components in principle also help in identifying the symmetry of the defect, in practice polarization measurements are often poor (particularly in PL measurements due to multiple depolarizing internal reflections). Even the absolute intensities can often be confusing, due to various other stress-induced effects which cause the component intensities to change, such as coupling with higher excited states, and exciton debinding/disassociation at high stresses [8]

In this report, we show that the application of a uniaxial stress on a single sample that is oriented along a crystal direction of very low symmetry, such as (136) for example, removes all orientational and electronic degeneracy for all transitions that can arise in a host lattice of cubic symmetry. We suggest that the use of the LSAPS technique in conjunction with traditional stress measurements will be helpful in clarifying cases of possible ambiguity by allowing complete removal of all degeneracy (both orientational and electronic). The effects of an arbitrary low-symmetry stress on defects in cubic crystals are given in the absence of inter-level interactions for all defect classes.

\section{Experiment}

We decided to use the so-called $\mathrm{Cd}_{A}$ defect in Czochralski (CZ) silicon as a test case for the LSAPS technique as this case is reasonably well characterized by traditional stress methods. $\mathrm{Si}: \mathrm{Cd}$ samples were produced by cadmium ion implantation into high-resistivity $\mathrm{CZ}$ material as described in more detail by McGlynn et al [8]. The samples were RCA cleaned and annealed in a clean quartz tube for a period of 30 minutes at $550{ }^{\circ} \mathrm{C}$. Samples were cut into rectangular parallelepipeds (with approximate dimensions $10 \mathrm{~mm} \times 3 \mathrm{~mm} \times 1.5 \mathrm{~mm}$ ), with the sample long axis nominally parallel to the (136) crystal axes while the other sides were along $(31-1)$ - and $(9-198)$-type axes. These samples were supplied to us by Virginia Semiconductor Corporation. The luminescence was excited using the $514 \mathrm{~nm}$ line of an argon-ion laser, and the excitation power was typically $\sim 200 \mathrm{~mW}$ (unfocused) on the sample face and the data were recorded using a Bomem DA8 FTIR spectrometer, coupled to an $\mathrm{LN}_{2}$ cooled North Coast germanium detector model EO-817 ED2. An Oxford Instruments CF1204 helium flow cryostat was used for low-temperature uniaxial stress experiments. The details of the apparatus used for the uniaxial stress measurements are described elsewhere [6]. 


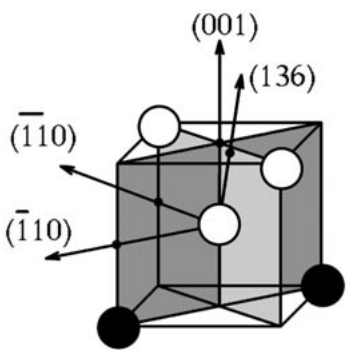

(a)

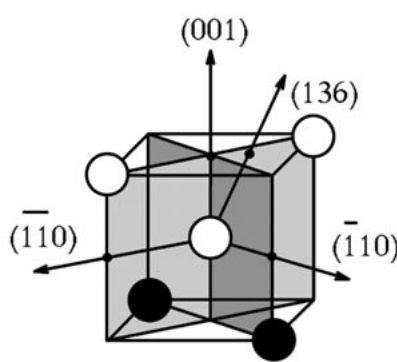

(b)

Figure 1. (a) A schematic representation of a $C_{2 v}$ symmetry defect within a $T_{d}$ lattice showing the $(001)$ rotation axis, (1 10$)$-type reflection planes and the (136) stress direction. (b) Similar to (a) but rotated through $90^{\circ}$ about the $(001)$ axis.

\section{Theory}

\subsection{Defects with orientational degeneracy}

Defects in crystals of cubic lattice symmetry such as silicon can possess one of several possible symmetry configurations: tetrahedral $\left(\mathrm{T}_{\mathrm{d}}\right)$, tetragonal $\left(\mathrm{D}_{2 \mathrm{~d}}\right)$, trigonal $\left(\mathrm{C}_{3 \mathrm{v}}\right)$, rhombic $\mathrm{I}\left(\mathrm{C}_{2 \mathrm{v}}\right)$, rhombic II $\left(\mathrm{D}_{2}\right)$, monoclinic I $\left(\mathrm{C}_{1 \mathrm{~h}}\right)$, monoclinic II $\left(\mathrm{C}_{2}\right)$ or triclinic $\left(\mathrm{C}_{1}\right)$. In this section we will only consider optical transitions occurring between levels possessing no electronic degeneracy. Uniaxial stress perturbations along any one of the (1 111$),\left(\begin{array}{lll}1 & 1 & 0\end{array}\right)$, or $\left(\begin{array}{lll}0 & 0 & 1\end{array}\right)$ directions will not remove all the orientational degeneracy of transitions between levels occurring about axial defects possessing any of these symmetry subgroups. It is for this reason that the accepted practice is to obtain three sets of uniaxial stress data corresponding to stress applied along each of the (1 111$),\left(\begin{array}{lll}1 & 1 & 0\end{array}\right)$ and $\left(\begin{array}{lll}0 & 0 & 1\end{array}\right)$ directions at different times. In principle this overspecifies the defect symmetry, with more stress components than unknown stress parameters, allowing a check of the quality of fit. The inability of these stresses to remove all the orientational degeneracy of transitions originating from defects with the symmetries listed earlier arises primarily from the fact that the unit vectors defining these three major crystal directions all have equal magnitude in the $x$ and $y$ directions. This, in turn, stems from the fact that the (1 111$),\left(\begin{array}{lll}1 & 1 & 0\end{array}\right)$ and $\left(\begin{array}{lll}0 & 0 & 1\end{array}\right)$ directions all lie in the [1 110$]$ plane. Consequently the components of any applied stress along the $x$ and $y$ directions for any of these defect symmetries are always identical.

In order to remove as much orientational degeneracy as possible we could instead apply uniaxial stress along a crystal direction $(X Y Z)$ such that:

$$
|X| \neq|Y| \neq|Z|
$$

and

$$
|X-Y| \neq|Y-Z| \neq|Z-X| .
$$

For example, the direction (136) would satisfy these conditions. For the sake of brevity we show how stress applied along this direction would affect an optical transition between electronically non-degenerate levels originating about a defect of rhombic $I\left(C_{2 v}\right)$ symmetry and then list the results, calculated in a similar manner for the other six subgroups of $T_{d}$. 
The symmetry operations of the $\mathrm{C}_{2 \mathrm{v}}$ point group are the identity, one $\mathrm{C}_{2}$ rotation and two $\sigma$ reflections in planes parallel to the $C_{2}$ axis [5]. Two schematic views of a $C_{2 v}$ symmetry defect are shown in figures 1 (a) and (b). The defect possesses a $\mathrm{C}_{2}$ rotation axis along the $\left(\begin{array}{lll}0 & 0 & 1\end{array}\right)$ direction, and two $\sigma_{\mathrm{v}}\left(\begin{array}{lll}1 & 1 & 0\end{array}\right)$-type reflection planes. The smaller black circles lying on each vector denote their point of exit from the defect unit cell. The (136) stress direction is clearly visible and is observed to lie outside all axes and planes of symmetry.

We define three mutually orthogonal, local (or defect) axes $X, Y$ and $Z$ to characterize each of the six inequivalent defect configurations of the rhombic I defect.

We define the matrix $T_{d}$ such that:

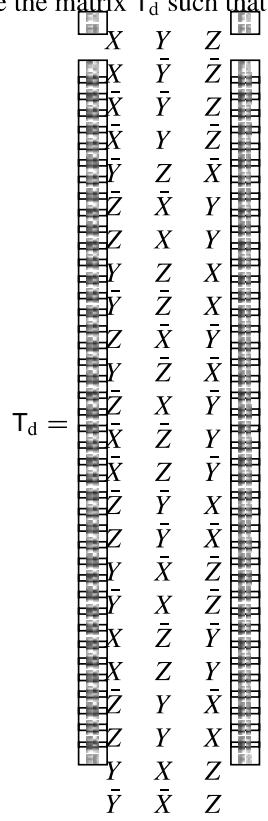

The 24 rows in this matrix correspond to the 24 inequivalent ( $X Y Z$ )-type axes that are possible for a $T_{d}$ crystal. We define the crystal axes $x, y$ and $z$ such that:

$$
\begin{aligned}
& x=\left(\begin{array}{lll}
1 & 0 & 0
\end{array}\right) \\
& y=\left(\begin{array}{lll}
0 & 1 & 0
\end{array}\right) \\
& z=\left(\begin{array}{lll}
0 & 0 & 1
\end{array}\right) .
\end{aligned}
$$

The strain tensor $S_{i j}$ is defined as:

$$
S_{i j}=|\mathrm{S}| \cos (\mathrm{S}, i) \cos (\mathrm{S}, j) \quad i, j \in x, y, z
$$

where $\cos (\mathrm{S}, i)$ is the cosine of the angle between the direction of the stress vector $\mathrm{S}$ and the $i$ th defect axis. The $S_{i j}$ value for each inequivalent ( $X Y Z$ )-type direction can be calculated as follows:

$$
\begin{aligned}
& \mathrm{S}_{x}=\mathrm{T}_{\mathrm{d}} * \mathrm{x} \\
& \mathrm{S}_{y}=\mathrm{T}_{\mathrm{d}} * \mathrm{y} \\
& \mathrm{S}_{z}=\mathrm{T}_{\mathrm{d}} * \mathrm{z} .
\end{aligned}
$$


Table 1. Stress potentials for electronically non-degenerate defects.

\begin{tabular}{ll}
\hline Group & Stress potential \\
\hline $\mathrm{T}_{\mathrm{d}}$ & $A_{1}\left(S_{x x}+S_{y y}+S_{z z}\right)$ \\
$\mathrm{D}_{2 \mathrm{~d}}$ & $A_{1} S_{z z}+A_{2}\left(S_{x x}+S_{y y}\right)$ \\
$\mathrm{C}_{3 \mathrm{v}}$ & $A_{1}\left(S_{x x}+S_{y y}+S_{z z}\right)+2 A_{2}\left(S_{x y}+S_{y z}+S_{z x}\right)$ \\
$\mathrm{C}_{2 \mathrm{v}}$ & $A_{1} S_{z z}+A_{2}\left(S_{x x}+S_{y y}\right)+2 A_{3} S_{x y}$ \\
$\mathrm{D}_{2}$ & $A_{1} S_{x x}+A_{2} S_{y y}+A_{3} S_{z z}$ \\
$\mathrm{C}_{1 \mathrm{~h}}$ & $A_{1} S_{z z}+A_{2}\left(S_{x x}+S_{y y}\right)+2 A_{3} S_{x y}+2 A_{4}\left(S_{y z}-S_{z x}\right)$ \\
$\mathrm{C}_{2}$ & $A_{1} S_{z z}+A_{2} S_{x x}+A_{3} S_{y y}+2 A_{4} S_{x y}$ \\
$\mathrm{C}_{1}$ & $A_{1} S_{z z}+A_{2} S_{x x}+A_{3} S_{y y}+2 A_{4} S_{x y}+2 A_{5} S_{y z}+2 A_{6} S_{z x}$ \\
\hline
\end{tabular}

The $S_{i j}$ stress tensor is calculated from the array product [7] (denoted by '*') of the $\mathrm{S}_{i}$ and $\mathrm{S}_{j}$ matrices, i.e.

$$
S_{i j}=\mathrm{S}_{i} * \mathrm{~S}_{j} \quad i, j \in x, y, z .
$$

Using the theory of Kaplyanskii [1] and crystal coordinates we note that the action of the applied stress on a defect of $\mathrm{C}_{2 \mathrm{v}}$ symmetry can be modelled using the stress potential $\mathrm{V}$ given by:

$$
\mathrm{V}=A_{1} S_{z z}+A_{2}\left(S_{x x}+S_{y y}\right)+2 A_{3} S_{x y}
$$

The stress potentials for transitions occurring at all electronically non-degenerate transitions in a $\mathrm{T}_{\mathrm{d}}$ host crystal are given [1] in table 1 . The $A_{i}$ values are characteristic of each defect. $\mathrm{V}$ will be a $24 \times 1$ column matrix, each row of which corresponds to the shift rate induced at a defect by each of the 24 inequivalent ( $X Y Z$ ) stress directions in a $\mathrm{T}_{\mathrm{d}}$ host crystal. For the case of an optical transition at a defect of $\mathrm{C}_{2 \mathrm{v}}$ symmetry, the zero-stress PL line will split into six components under stress, each with shift rate $R_{i}(i \in 1$ to 6$)$ :

$$
\begin{aligned}
& R_{1}=A_{1} X^{2}+A_{2}\left(Y^{2}+Z^{2}\right)+2 A_{3} Y Z \\
& R_{2}=A_{1} X^{2}+A_{2}\left(Y^{2}+Z^{2}\right)-2 A_{3} Y Z \\
& R_{3}=A_{1} Y^{2}+A_{2}\left(X^{2}+Z^{2}\right)+2 A_{3} Z X \\
& R_{4}=A_{1} Y^{2}+A_{2}\left(X^{2}+Z^{2}\right)-2 A_{3} Z X \\
& R_{5}=A_{1} Z^{2}+A_{2}\left(X^{2}+Y^{2}\right)+2 A_{3} X Y \\
& R_{6}=A_{1} Z^{2}+A_{2}\left(X^{2}+Y^{2}\right)-2 A_{3} X Y .
\end{aligned}
$$

Consequently we can see that uniaxial stress applied along the $(X Y Z)$ crystal direction removes all orientational degeneracy, if $X, Y$ and $Z$ satisfy the conditions laid out in equations (1) and (2).

The theoretical shift rate equations for all orientationally degenerate but electronically non-degenerate transitions in a $T_{d}$ symmetry host crystal, calculated by similar means, are given in tables 2 and 3 .

\subsection{Defects with electronic degeneracy}

For the case of defects with sufficiently high symmetry to possess electronic as well as orientational degeneracy, such as trigonal, tetragonal and tetrahedral defects, the argument is only slightly more complicated. Just as in the case of the non-degenerate states described above, the 24 inequivalent directions are described by the matrix $T_{d}$ of equation (3). The stress tensor $S_{i j}$ is calculated as in equations (8)-(11). The difference for electronically degenerate defects arises from the fact that the action of the applied stress on the electronically degenerate 
Table 2. Shift rate equations for non-degenerate defect states under unit stress along the ( $X Y Z)$ direction.

\begin{tabular}{|c|c|}
\hline Transition & Shift rate equation ${ }^{\mathrm{a}}$ \\
\hline $\begin{array}{l}\text { Triclinic } \\
\left(\mathrm{C}_{1}\right) \\
\mathrm{A} \text { to } \mathrm{A}\end{array}$ & $\begin{array}{l}A_{1} X^{2}+A_{2} Y^{2}+A_{3} Z^{2}+2 A_{4} X Y+2 A_{5} Y Z+2 A_{6} Z X \\
A_{1} X^{2}+A_{2} Y^{2}+A_{3} Z^{2}-2 A_{4} X Y+2 A_{5} Y Z-2 A_{6} Z X \\
A_{1} X^{2}+A_{2} Y^{2}+A_{3} Z^{2}+2 A_{4} X Y-2 A_{5} Y Z-2 A_{6} Z X \\
A_{1} X^{2}+A_{2} Y^{2}+A_{3} Z^{2}-2 A_{4} X Y-2 A_{5} Y Z+2 A_{6} Z X \\
A_{1} X^{2}+A_{2} Z^{2}+A_{3} Y^{2}+2 A_{4} Z X+2 A_{5} Y Z+2 A_{6} X Y \\
A_{1} X^{2}+A_{2} Z^{2}+A_{3} Y^{2}-2 A_{4} Z X+2 A_{5} Y Z-2 A_{6} X Y \\
A_{1} X^{2}+A_{2} Z^{2}+A_{3} Y^{2}+2 A_{4} Z X-2 A_{5} Y Z-2 A_{6} X Y \\
A_{1} X^{2}+A_{2} Z^{2}+A_{3} Y^{2}-2 A_{4} Z X-2 A_{5} Y Z+2 A_{6} X Y \\
A_{1} Y^{2}+A_{2} X^{2}+A_{3} Z^{2}+2 A_{4} X Y+2 A_{5} Z X+2 A_{6} Y Z \\
A_{1} Y^{2}+A_{2} X^{2}+A_{3} Z^{2}-2 A_{4} X Y+2 A_{5} Z X-2 A_{6} Y Z \\
A_{1} Y^{2}+A_{2} X^{2}+A_{3} Z^{2}+2 A_{4} X Y-2 A_{5} Z X-2 A_{6} Y Z \\
A_{1} Y^{2}+A_{2} X^{2}+A_{3} Z^{2}-2 A_{4} X Y-2 A_{5} Z X+2 A_{6} Y Z \\
A_{1} Y^{2}+A_{2} Z^{2}+A_{3} X^{2}+2 A_{4} Y Z+2 A_{5} Z X+2 A_{6} X Y \\
A_{1} Y^{2}+A_{2} Z^{2}+A_{3} X^{2}-2 A_{4} Y Z+2 A_{5} Z X-2 A_{6} X Y \\
A_{1} Y^{2}+A_{2} Z^{2}+A_{3} X^{2}+2 A_{4} Y Z-2 A_{5} Z X-2 A_{6} X Y \\
A_{1} Y^{2}+A_{2} Z^{2}+A_{3} X^{2}-2 A_{4} Y Z-2 A_{5} Z X+2 A_{6} X Y \\
A_{1} Z^{2}+A_{2} X^{2}+A_{3} Y^{2}+2 A_{4} Z X+2 A_{5} X Y+2 A_{6} Y Z \\
A_{1} Z^{2}+A_{2} X^{2}+A_{3} Y^{2}-2 A_{4} Z X+2 A_{5} X Y-2 A_{6} Y Z \\
A_{1} Z^{2}+A_{2} X^{2}+A_{3} Y^{2}+2 A_{4} Z X-2 A_{5} X Y-2 A_{6} Y Z \\
A_{1} Z^{2}+A_{2} X^{2}+A_{3} Y^{2}-2 A_{4} Z X-2 A_{5} X Y+2 A_{6} Y Z \\
A_{1} Z^{2}+A_{2} Y^{2}+A_{3} X^{2}+2 A_{4} Y Z+2 A_{5} X Y+2 A_{6} Z X \\
A_{1} Z^{2}+A_{2} Y^{2}+A_{3} X^{2}-2 A_{4} Y Z+2 A_{5} X Y-2 A_{6} Z X \\
A_{1} Z^{2}+A_{2} Y^{2}+A_{3} X^{2}+2 A_{4} Y Z-2 A_{5} X Y-2 A_{6} Z X \\
A_{1} Z^{2}+A_{2} Y^{2}+A_{3} X^{2}-2 A_{4} Y Z-2 A_{5} X Y+2 A_{6} Z X\end{array}$ \\
\hline $\begin{array}{l}\text { Monoclinic I } \\
\left(\mathrm{C}_{1 \mathrm{~h}}\right) \\
\mathrm{A}^{\prime} \text { to } \mathrm{A}^{\prime} \\
\mathrm{A}^{\prime \prime} \text { to } \mathrm{A}^{\prime \prime} \\
\mathrm{A}^{\prime} \text { to } \mathrm{A}^{\prime \prime}\end{array}$ & $\begin{array}{l}A_{1} X^{2}+A_{2}\left(Y^{2}+Z^{2}\right)+2 A_{3} Y Z+2 A_{4}(Z X-X Y) \\
A_{1} X^{2}+A_{2}\left(Y^{2}+Z^{2}\right)-2 A_{3} Y Z+2 A_{4}(Z X+X Y) \\
A_{1} X^{2}+A_{2}\left(Y^{2}+Z^{2}\right)+2 A_{3} Y Z-2 A_{4}(Z X-X Y) \\
A_{1} X^{2}+A_{2}\left(Y^{2}+Z^{2}\right)-2 A_{3} Y Z-2 A_{4}(Z X+X Y) \\
A_{1} Y^{2}+A_{2}\left(X^{2}+Z^{2}\right)+2 A_{3} Z X+2 A_{4}(X Y-Y Z) \\
A_{1} Y^{2}+A_{2}\left(X^{2}+Z^{2}\right)-2 A_{3} Z X+2 A_{4}(X Y+Y Z) \\
A_{1} Y^{2}+A_{2}\left(X^{2}+Z^{2}\right)+2 A_{3} Z X-2 A_{4}(X Y-Y Z) \\
A_{1} Y^{2}+A_{2}\left(X^{2}+Z^{2}\right)-2 A_{3} Z X-2 A_{4}(X Y+Y Z) \\
A_{1} Z^{2}+A_{2}\left(X^{2}+Y^{2}\right)+2 A_{3} X Y+2 A_{4}(Y Z-Z X) \\
A_{1} Z^{2}+A_{2}\left(X^{2}+Y^{2}\right)-2 A_{3} X Y+2 A_{4}(Y Z+Z X) \\
A_{1} Z^{2}+A_{2}\left(X^{2}+Y^{2}\right)+2 A_{3} X Y-2 A_{4}(Y Z-Z X) \\
A_{1} Z^{2}+A_{2}\left(X^{2}+Y^{2}\right)-2 A_{3} X Y-2 A_{4}(Y Z+Z X)\end{array}$ \\
\hline $\begin{array}{l}\text { Monoclinic II } \\
\left(\mathrm{C}_{2}\right) \\
\text { A to } A \\
\text { A to } B \\
\text { B to } B^{\prime}\end{array}$ & $\begin{array}{l}A_{1} X^{2}+A_{2} Y^{2}+A_{3} Z^{2}+2 A_{4} Y Z \\
A_{1} X^{2}+A_{2} Y^{2}+A_{3} Z^{2}-2 A_{4} Y Z \\
A_{1} X^{2}+A_{2} Z^{2}+A_{3} Y^{2}+2 A_{4} Y Z \\
A_{1} X^{2}+A_{2} Z^{2}+A_{3} Y^{2}-2 A_{4} Y Z \\
A_{1} Y^{2}+A_{2} X^{2}+A_{3} Z^{2}+2 A_{4} Z X \\
A_{1} Y^{2}+A_{2} X^{2}+A_{3} Z^{2}-2 A_{4} Z X \\
A_{1} Y^{2}+A_{2} Z^{2}+A_{3} X^{2}+2 A_{4} Z X \\
A_{1} Y^{2}+A_{2} Z^{2}+A_{3} X^{2}-2 A_{4} Z X \\
A_{1} Z^{2}+A_{2} X^{2}+A_{3} Y^{2}+2 A_{4} X Y \\
A_{1} Z^{2}+A_{2} X^{2}+A_{3} Y^{2}-2 A_{4} X Y \\
A_{1} Z^{2}+A_{2} Y^{2}+A_{3} X^{2}+2 A_{4} X Y \\
A_{1} Z^{2}+A_{2} Y^{2}+A_{3} X^{2}+2 A_{4} X Y\end{array}$ \\
\hline
\end{tabular}

${ }^{\text {a }}$ N.B. All equations should be divided by $\left(X^{2}+Y^{2}+Z^{2}\right)$. 
Table 3. Shift rate equations for non-degenerate defect states under unit stress along the ( $X Y Z)$ direction.

\begin{tabular}{ll}
\hline Transition & Shift rate equation \\
\hline Tetragonal & $A_{1} X^{2}+A_{2}\left(Y^{2}+Z^{2}\right)$ \\
$\left(\mathrm{D}_{2 \mathrm{~d}}\right)$ & $A_{1} Y^{2}+A_{2}\left(X^{2}+Z^{2}\right)$ \\
A to B & $A_{1} Z^{2}+A_{2}\left(X^{2}+Y^{2}\right)$ \\
Trigonal & $A_{1}\left(X^{2}+Y^{2}+Z^{2}\right)+2 A_{2}(X Y+Y Z+Z X)$ \\
(C $\left.\mathrm{C}_{3 \mathrm{v}}\right)$ & $A_{1}\left(X^{2}+Y^{2}+Z^{2}\right)-2 A_{2}(X Y-Y Z+Z X)$ \\
A to A & $A_{1}\left(X^{2}+Y^{2}+Z^{2}\right)+2 A_{2}(X Y-Y Z-Z X)$ \\
& $A_{1}\left(X^{2}+Y^{2}+Z^{2}\right)-2 A_{2}(X Y+Y Z-Z X)$ \\
Rhombic I & $A_{1} X^{2}+A_{2}\left(Y^{2}+Z^{2}\right)+2 A_{3} Y Z$ \\
(C $\left.\mathrm{C}_{2 \mathrm{v}}\right)$ & $A_{1} X^{2}+A_{2}\left(Y^{2}+Z^{2}\right)-2 A_{3} Y Z$ \\
A to A & $A_{1} Y^{2}+A_{2}\left(X^{2}+Z^{2}\right)+2 A_{3} Z X$ \\
A to B & $A_{1} Y^{2}+A_{2}\left(X^{2}+Z^{2}\right)-2 A_{3} Z X$ \\
B to B & $A_{1} Z^{2}+A_{2}\left(X^{2}+Y^{2}\right)+2 A_{3} X Y$ \\
& $A_{1} Z^{2}+A_{2}\left(X^{2}+Y^{2}\right)-2 A_{3} X Y$ \\
Rhombic II & $A_{1} X^{2}+A_{2} Y^{2}+A_{3} Z^{2}$ \\
(D $\left.{ }_{2}\right)$ & $A_{1} X^{2}+A_{2} Z^{2}+A_{3} Y^{2}$ \\
A to B & $A_{1} Y^{2}+A_{2} X^{2}+A_{3} Z^{2}$ \\
& $A_{1} Y^{2}+A_{2} Z^{2}+A_{3} X^{2}$ \\
& $A_{1} Z^{2}+A_{2} X^{2}+A_{3} Y^{2}$ \\
& $A_{1} Z^{2}+A_{2} Y^{2}+A_{3} X^{2}$ \\
\hline
\end{tabular}

a N.B. All equations should be divided by $\left(X^{2}+Y^{2}+Z^{2}\right)$.

defect state is now modelled by diagonalizing the matrix $\mathrm{VW}^{*}$, which, for example at a doubly degenerate (E) state at a trigonal defect, is given by [9]

$$
\mathrm{VW}^{*}=\begin{array}{cc}
\alpha-\beta & \gamma \\
\gamma & \alpha+\beta
\end{array}
$$

where

$$
\begin{aligned}
& \alpha=A_{1}\left(S_{x x}+S_{y y}+S_{z z}\right)+2 A_{2}\left(S_{y z}+S_{z x}+S_{x y}\right) \\
& \beta=B\left(2 S_{z z}-S_{y y}-S_{x x}\right)+C\left(2 S_{x y}-S_{y z}-S_{z x}\right) \\
& \gamma=\sqrt{3} B\left(S_{x x}-S_{y y}\right)+\sqrt{3} C\left(S_{y z}-S_{z x}\right) .
\end{aligned}
$$

$B$ and $C$ are the matrix elements of the stress operators between the $\mathrm{E}_{x}$ and $\mathrm{E}_{y}$ basis states [10] and characterize the defect identity in the same manner as $A_{1}$ and $A_{2}$. Thus in the case of electronic degeneracy, the 24 inequivalent directions give rise to 24 matrices $\mathrm{VW}^{*}$, which give the shift rates corresponding to the lifting of both electronic and orientational degeneracies when diagonalized. For example, in the case of an E state at a trigonal defect, the optical transition will split into eight different components (four inequivalent orientations of the defect with two electronic levels per orientation) as given in table 4 . The splitting patterns for transitions between a non-degenerate ground state and all electronically degenerate states at trigonal, tetragonal and tetrahedral defects are given in tables 4-6.

\subsection{Additional comments}

We have not calculated intensity information for any of the stress sub-components listed in tables $2-4$, nor do we make but a passing reference to them in sections 4 and 5 , for a 
Table 4. Shift rate equations for trigonal $\mathrm{E}$ states under unit stress along the $(X Y Z)$ direction.

\begin{tabular}{|c|c|c|c|c|}
\hline \multirow{5}{*}{$\begin{array}{l}\text { Interacti } \\
\text { matrix } \\
\begin{array}{c}\alpha-\beta \\
\gamma\end{array}\end{array}$} & & $\begin{array}{l}\text { General shift } \\
\text { rate equation }\end{array}$ & & $\begin{array}{l}\text { Inequivalent stress axis and } \\
\text { shift rate equation components }{ }^{\mathrm{a}}\end{array}$ \\
\hline & \multirow[t]{4}{*}{$\begin{array}{c}\gamma \\
\alpha+\beta\end{array}$} & \multirow[t]{4}{*}{$\alpha \pm \beta^{2}+\gamma^{2}$} & $X Y Z$ & $\begin{array}{l}\alpha=A_{1}\left(X^{2}+Y^{2}+Z^{2}\right)+2 A_{2}(X Y+Y Z+Z X) \\
\beta=B\left(2 Z^{2}-X^{2}-Y^{2}\right)+C(2 X Y-Y Z-Z X) \\
\gamma=\sqrt{3} B\left(X^{2}-Y^{2}\right)+\sqrt{3} C(Y Z-Z X)\end{array}$ \\
\hline & & & $\overline{X Y} Z$ & $\begin{aligned} \alpha & =A_{1}\left(X^{2}+Y^{2}+Z^{2}\right)+2 A_{2}(X Y-Y Z-Z X) \\
\beta & =B\left(2 Z^{2}-X^{2}-Y^{2}\right)+C(2 X Y+Y Z+Z X) \\
\gamma & =\sqrt{3} B\left(X^{2}-Y^{2}\right)+\sqrt{3} C(Y Z-Z X)\end{aligned}$ \\
\hline & & & $\bar{X} Y \bar{Z}$ & $\begin{array}{l}\alpha=A_{1}\left(X^{2}+Y^{2}+Z^{2}\right)-2 A_{2}(X Y-Y Z+Z X) \\
\beta=B\left(2 Z^{2}-X^{2}-Y^{2}\right)-C(2 X Y+Y Z-Z X) \\
\gamma=\sqrt{3} B\left(Y^{2}-X^{2}\right)+\sqrt{3} C(Y Z+Z X)\end{array}$ \\
\hline & & & $X \overline{Y Z}$ & $\begin{array}{l}\alpha=A_{1}\left(X^{2}+Y^{2}+Z^{2}\right)-2 A_{2}(X Y+Y Z-Z X) \\
\beta=B\left(2 Z^{2}-X^{2}-Y^{2}\right)+C(2 X Y-Y Z+Z X) \\
\gamma=\sqrt{3} B\left(Y^{2}-X^{2}\right)+\sqrt{3} C(Y Z+Z X)\end{array}$ \\
\hline
\end{tabular}

${ }^{a}$ N.B. All equations should be divided by $\left(X^{2}+Y^{2}+Z^{2}\right)$.

Table 5. Shift rate equations for tetragonal $\mathrm{E}$ states under unit stress along the $(X Y Z)$ direction.

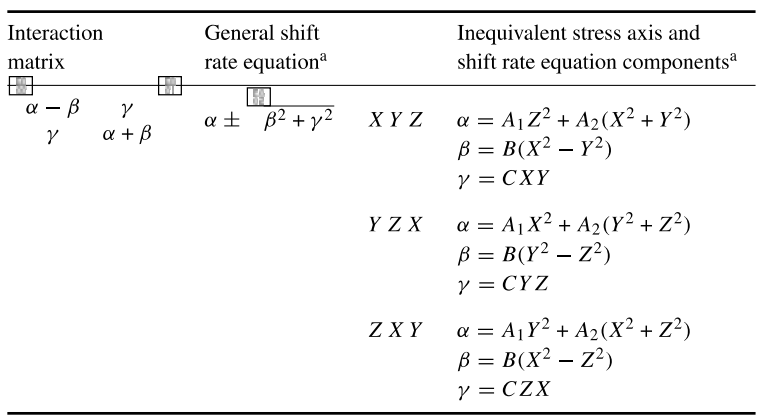

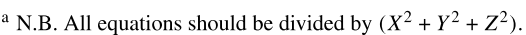

number of reasons. Firstly, even in the case of electronically non-degenerate defects (for which the polarized intensities may be calculated relatively easily if the orientations of the three crystal faces are known [1,2]), for an arbitrary low-symmetry stress direction, the crystal becomes bi-axial. Consequently the perpendicular polarizations depend on the choice of viewing direction, for which there is no natural choice in the case of LSAPS (unlike a $\left(\begin{array}{lll}1 & 1 & 0\end{array}\right)$ stress, where the $\left(\begin{array}{lll}0 & 0 & 1\end{array}\right)$ and $\left(\begin{array}{lll}1 & 1 & 0\end{array}\right)$ directions suggest themselves naturally). In the case of electronically degenerate states, the problem is more fundamental. The matrix elements in $\mathrm{VW}^{*}$ are functions of stress; hence the composition of the eigenfunctions (and transition probability) changes with stress for low-symmetry-axis stress directions [9]. Secondly, we propose the LSAPS technique as a supplement to the traditional stress methods, which will have greatest benefit in distinguishing between various possibilities which cannot be resolved via the normal technique, rather than as a stand-alone method. In these cases the most important considerations in resolving the confusion will be knowledge of the number of components and their shift rates. 
Table 6. Shift rate equations for tetrahedral $\mathrm{E}, \mathrm{T}$ and $\Gamma_{8}$ states under unit stress along the ( $\left.X Y Z\right)$ direction are the eigenvalue equations of the corresponding interaction matrix.

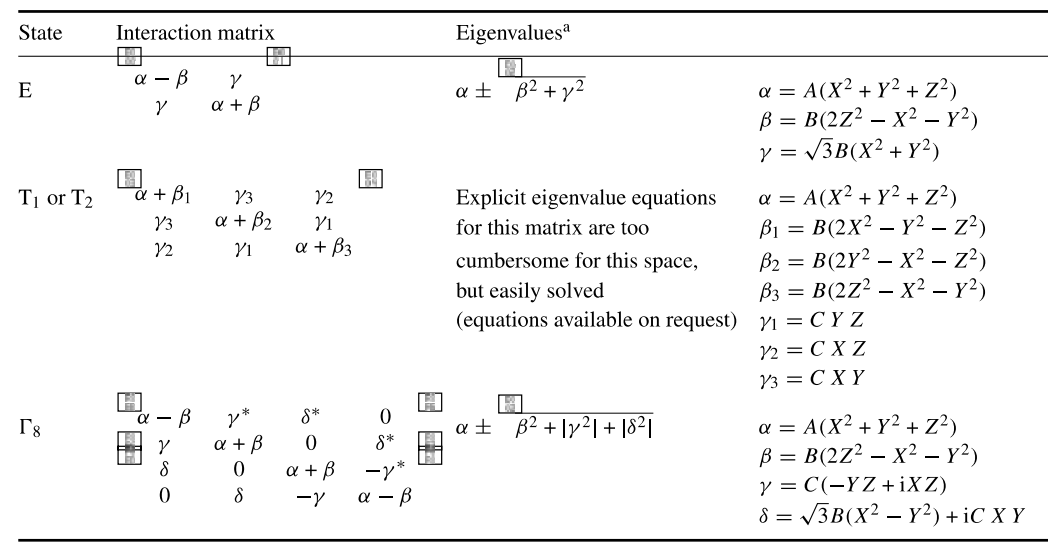

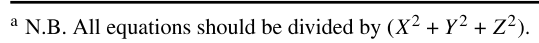

It is also appropriate to comment at this point that the LSAPS technique will only be possible in high-quality materials systems (such as $\mathrm{Si}, \mathrm{Ge}, \mathrm{C}, \mathrm{GaAs}$ etc) where the narrow line widths of optical transitions will in principle allow the relatively large number of subcomponents produced by the LSAPS to be resolved. Even in the situation where all components are not fully resolved however, it remains the case that the technique may successfully distinguish between various possibilities by placing lower bounds on the number of possible components.

\section{Results}

4.1. Theoretical validation

The validity of the theory used to evaluate the low-symmetry-axis perturbation spectroscopy (LSAPS) effects was confirmed by using it to successfully predict the number of stress induced components and their shift rates for all transitions occurring about defects with symmetries that are sub-groups of $\mathrm{T}_{\mathrm{d}}$ under (1 111$),\left(\begin{array}{lll}1 & 1 & 0\end{array}\right)$ and (l0 01 ) stresses, as reported by Kaplyanskii [1,2] and Mohammed et al [3].

\subsection{Experimental validation $-\mathrm{Cd}_{A}\left(C_{2 v}\right)$ system in silicon}

Uniaxial stress was applied to an Si:Cd sample, oriented nominally along the (136) crystal direction. The stress induced splitting of the $983.21 \mathrm{MeV} \mathrm{Cd}_{A}$ [8] zero-phonon PL transition was observed. Previous studies [8] have identified this PL system as possessing rhombic I $\mathrm{C}_{2 \mathrm{v}}$ symmetry. In figures 2 and 3 we see that the line splits into six stress induced components (numbered $L_{1}$ to $L_{6}$ in ascending order of line energy) consistent with the $\mathrm{C}_{2 \mathrm{v}}$ defect symmetry reported previously [8]. The solid lines in figure 3 are fits to the theoretical shift rate equations listed in table 3 for a $C_{2 v}$ defect for $X=1, Y=3, Z=6, A_{1}=-46.3 \mathrm{MeV} \mathrm{GPa}^{-1}$, $A_{2}=12.88 \mathrm{MeV} \mathrm{GPa}^{-1}$ and $A_{3}=-17.55 \mathrm{MeV} \mathrm{GPa}^{-1}$. These $A_{i}$ values are in good agreement with the values found previously [8] (see table 7). 


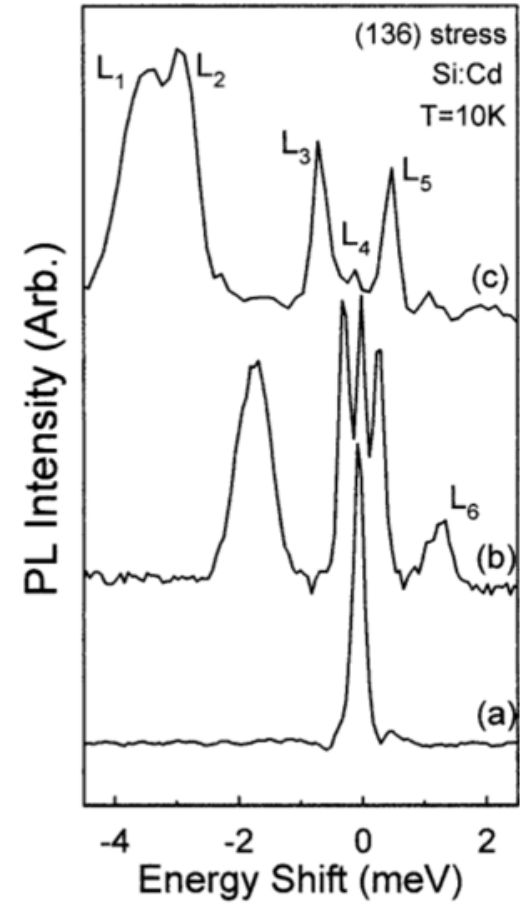

Figure 2. Representative spectra at $T=10 \mathrm{~K}$ of the $983.21 \mathrm{MeV} \mathrm{Cd}_{A}$ line in silicon [8] under stress along the (136) direction at (a) $0 \mathrm{MPa}$, (b) $47 \mathrm{MPa}$ and (c) $89 \mathrm{MPa}$

We note that the intensities of the stress sub-components are not constant as a function of stress, and in fact the components $\mathrm{L}_{3}, \mathrm{~L}_{4}, \mathrm{~L}_{5}$ and $\mathrm{L}_{6}$ all reduce in intensity compared to $\mathrm{L}_{1}$ and $\mathrm{L}_{2}$. The lines $\mathrm{L}_{4}$ and $\mathrm{L}_{6}$ show the largest reduction in intensity, whereas $\mathrm{L}_{3}$ and $\mathrm{L}_{5}$ show a smaller reduction in intensity as stress increases. This behaviour has been seen for the $\mathrm{Cd}_{A}$ defect previously [8], where a reduction in intensity of certain sub-components was observed (most noticeably under $\left\langle\begin{array}{llll}0 & 0 & 1\end{array}\right\rangle$ stress). This was attributed to a stress induced change in the ability of the orientationally inequivalent centres to bind one or both of the excitonic particles at the defect, and it may be shown that the effect is most pronounced for defects whose principal $\left(\mathrm{C}_{2}\right)$ axis is orthogonal to the stress direction. The reduction in intensity observed here is in agreement with the previous measurements. The pair of lines showing the strongest reduction in intensity $\left(\mathrm{L}_{4}\right.$ and $\left.\mathrm{L}_{6}\right)$ corresponds to defect orientations whose principal axes are oriented at an angle of $81^{\circ}$ to the nominal $\langle 136\rangle$ stress direction. $\mathrm{L}_{3}$ and $\mathrm{L}_{5}$ have their principal axes oriented at $64^{\circ}$ to the stress axis, and $\mathrm{L}_{1}$ and $\mathrm{L}_{2}$ have their principal axes oriented at $28^{\circ}$ to the stress direction. Thus as the defect principal axis makes a larger angle to the stress direction, the line intensity reduces in agreement with the observations in [8]. 


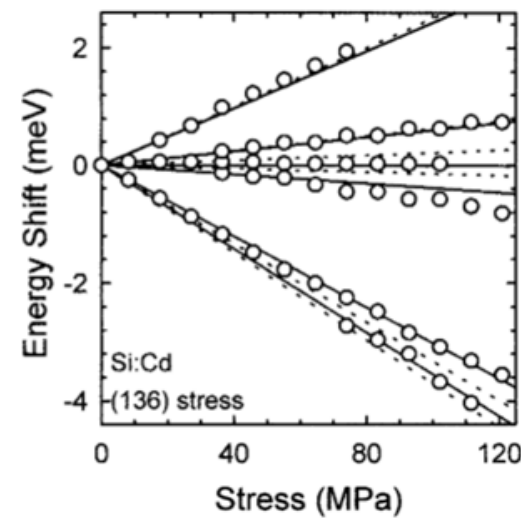

Figure 3. Fan diagram showing the stress induced splitting of the $983.21 \mathrm{MeV} \mathrm{Cd}_{A}$ line in silicon [8] under (136) stress. Dashed lines represent theoretical fits using the $A_{i}$ values reported by McGlynn et al [8] and stress along (136). Solid lines assume stress along (143361), i.e. a misalignment of $3.4^{\circ}$ away from (136)

Table 7. Comparisons of theoretical fits to the experimental data for (136) stress on the $983.21 \mathrm{MeV}$ $\mathrm{Cd}_{A}$ PL line in silicon.

\begin{tabular}{lclc}
\hline $\begin{array}{l}\text { Measured } \\
\text { shift rates } \\
\left(\mathrm{meV} \mathrm{GPa}^{-1}\right)\end{array}$ & $\begin{array}{l}\text { LSAPS (136) theoretical } \\
\text { best-fit shift rates } \\
\left(\mathrm{meV} \mathrm{GPa}^{-1}\right)\end{array}$ & $\begin{array}{l}\text { McGlynn et al (136) } \\
\text { predicted shift rates }^{\mathrm{a}} \\
\left(\mathrm{meV} \mathrm{GPa}^{-1}\right)\end{array}$ & $\begin{array}{l}\text { McGlynn et al (143361) } \\
\text { theoretical shift rates } \\
\left(\mathrm{meV} \mathrm{GPa}^{\mathrm{b}}\right)\end{array}$ \\
\hline$-35.9 \pm 2.8$ & -35.7 & -37.1 & -35.5 \\
$-30.2 \pm 1.6$ & -31.1 & -33.3 & -30.2 \\
$-7.0 \pm 1.5$ & -3.3 & -1.4 & -3.8 \\
$-0.1 \pm 2.8$ & -2.1 & 2.2 & -0.1 \\
$6.2 \pm 1.8$ & 5.9 & 6.2 & 6.0 \\
$25.9 \pm 2.4$ & 25.3 & 25.0 & 24.3 \\
Fitted stress parameters (meV GPa & & \\
$A_{1}$ & -46.3 & -49.2 & -49.2 \\
$A_{2}$ & 12.8 & 15.0 & 15.0 \\
$A_{3}$ & -17.5 & -14.6 & -14.6 \\
Least square fit error (Arb.) & 7.7 & 13.2 & 6.1 \\
\hline
\end{tabular}

a Dashed lines in figure 2

${ }^{\mathrm{b}}$ Solid lines in figure 2 .

Finally, we note that the intensities of the components at low stresses are in reasonable agreement with those expected from an analysis of this particular crystal orientation.

\section{Discussion}

From a general viewpoint, in the absence of polarization information, matching the stress induced components observed in the stress spectra with the correct theoretical shift rate from table 3 can be problematical. However, assigning the appropriate shift rate equation to its corresponding spectral component can be accomplished in the following manner. 
The six theoretical shift rate equations $R_{i}$, for a defect of $\mathrm{C}_{2 \mathrm{v}}$ symmetry under uniaxial stress may be written as:

$$
R_{i}=m_{j=1} m_{i j} \quad \text { for } 1 \square[6 .
$$

The $A_{j}$ are constants characteristic of the defect. We construct a $6 \times 3$ matrix M whose entry in the $i$ th row and $j$ th column is given by $m_{i j}$. We denote this by $\mathrm{M}=\left(m_{i j}\right)_{i=1, j=1}^{6,3}$. From table 3 we see that in this case for $\mathrm{C}_{2 \mathrm{v}}$ symmetry and $(X Y Z)=(136)$ :

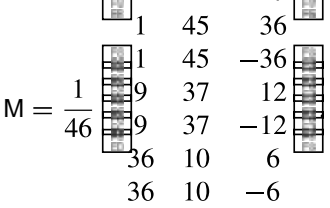

We define matrices $S_{\text {and }}$ a as follows:

$$
\begin{aligned}
& \mathrm{S}=\mathrm{H}_{2} \text {. 四 } \\
& \mathrm{A}=\begin{array}{l}
R_{6} \\
A_{1} \\
A_{2} \\
A_{3}
\end{array} .
\end{aligned}
$$

Thus we can write the shift rate equations as one matrix equation:

$$
\mathrm{S}=\mathrm{MA} \text {. }
$$

Experimentally we measure the six line shift rates $\left(\mathrm{L}_{i} i=1\right.$ to 6$)$ from the piezo-spectroscopic PL spectra. In the absence of polarization information, there are $6 ! \quad(=720)$ ways of substituting the six experimentally measured $\mathrm{L}_{i}$ for the six theoretical shift rates $R_{i}$. Denote

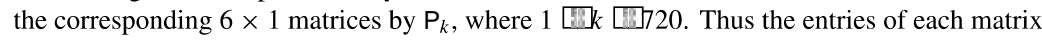
$\mathrm{P}_{k}$ will be the six $\mathrm{L}_{i}$ arranged in some order. There are 720 'ordered' ways of arranging the six $\mathrm{L}_{i}$ and so there will be $720 \mathrm{P}_{k}$ matrices.

We know the values of the entries in the matrices $\mathrm{P}_{k}$ and $\mathrm{M}$. We then obtain the best 'least squares fit' $F_{k}$ for the unknown matrix $\mathrm{A}$, for each of the 720 matrix equations $\mathrm{P}_{k}=\mathrm{MA}$. This is easily done with mathematics software packages such as Maple or Matlab [7]. Thus for each $k=1,2, \ldots, 720, F_{k}$ is the unique $3 \times 1$ matrix (from all $3 \times 1$ matrices) such that $\left\|\mathrm{P}_{k}-\mathrm{MF}_{k}\right\|$ takes on the smallest value, where $\left\|\mathrm{P}_{k}-\mathrm{MF}_{k}\right\|^{2}$ is the sum of the squares of all the entries in the matrix $\mathrm{P}_{k}-\mathrm{MF}_{k}$. The uniqueness above follows from the fact that the rank of the $6 \times 3$ matrix $M$ is 3 .

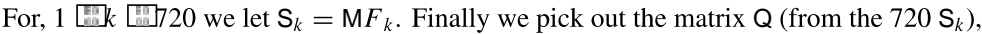
whose six entries are the closest to the $\operatorname{six~} \mathrm{L}_{i}$, in any order, in the sense of least squares. The six entries in $\mathrm{Q}$ give us our theoretical shift rate estimates for the experimentally measured shift rates $\mathrm{L}_{i}, 1$ [8] $\left[\begin{array}{ll}6 \\ 6\end{array}\right.$. The $R_{i}$ theoretical estimates listed in column 2 of table 7 are for the $\mathrm{Cd}_{A}$ system and the $A_{j}$ estimates come from the entries of $\mathrm{F}_{r}$ where $\mathrm{Q}=\mathrm{MF}_{r}$.

For defects in $\mathrm{T}_{\mathrm{d}}$ host crystals, the worst case scenario would be a triclinic defect where we have $24 R_{i}$ shift rate equations in six $A_{i}$ stress parameters (see table 2). In general, we have $m R_{i}$ with $n A_{i}, m>n$. We can still proceed as in the special case outlined above for $\mathrm{C}_{2 \mathrm{v}}$ defects where $\mathrm{M}$ will now be an $m \times n$ matrix and $\mathrm{A}$ will be an $n \times 1$ matrix. S will be an $m \times 1$ matrix. We find the 'least squares fit' $\mathrm{F}_{k}$ (for the unknown matrix $\mathrm{A}$ ) where $k=1,2,3, \ldots, n$ !. 
$\mathrm{F}_{k}$ will be unique if the rank of $\mathrm{M}$ is $n$. Otherwise (i.e. rank of $\mathrm{M}<n$ ) there will be infinitely many solutions to the least squares problem. We then proceed to find the $m \times 1$ matrix $\mathrm{Q}$ that will give us the theoretical shift rate estimates and check the errors between the theoretical shift rate estimates and the experimentally measured $\mathrm{L}_{i}$ shift rates. If the errors are within acceptable limits, we can therefore estimate the $A_{j}$ as we did in the special case.

From table 7 we see that the theoretical fits achieved using the procedure we have just described gives $A_{j}$ stress parameters that are in reasonable agreement with, and a lower least squares fit error than, those reported previously for the $\mathrm{Cd}_{A}$ and other rhombic I defects in silicon. In fact, both our results and those of McGlynn et al [8] can be fully reconciled if we assume that there is a misalignment of $3.4^{\circ}$ between the applied stress and the (136) crystal direction. Effectively this equates to a situation where the stress is applied along the (143361) crystal direction. Under such circumstances, using the $A_{i}$ values reported by McGlynn et al [8] the most accurate fit of all (solid lines in Figure 3) is accomplished (see column 4 of table 7). Some initial $x$-ray measurements of the sample orientation have been attempted, which confirm in general the orientation of the crystal as (136) to within $\sim 2^{\circ}$. We have not as yet performed sufficiently accurate measurements of the orientation to establish whether the misorientation of $3.4^{\circ}$ given by our best fit (with the sample axis along (143361)) is due to a combination of a slight misalignment during the production of the sample in addition to a slight misorientation of the sample during mounting in the stress cell, or entirely due to misorientation during mounting in the cell.

A major advantage of this technique is evident in situations where standard uniaxial stress analysis is unable to make unambiguous defect identification. The possible ambiguity between trigonal A-E transitions and transitions at a centre of monoclinic I symmetry has been discussed earlier. One may also appreciate that this technique will help in correctly identifying defect symmetries when an 'accidental degeneracy' remains in one of the components (due either to instrumental limitations and/or values of the parameters in the potential) under stress along one of the high-symmetry directions. In addition, since LSAPS removes all orientational degeneracy from a defect, it would be an ideal characterization technique for the study of re-orientation effects under stress.

\section{Conclusions}

Our calculations show that the application of a uniaxial stress along a crystal direction of low symmetry, such as the (136) direction, removes all orientational degeneracy from transitions arising from defects in tetrahedral crystals. We have demonstrated experimentally that the manifold of lines expected from a low-symmetry centre such as rhombic I may be successfully resolved and that the splitting pattern in this case is in excellent agreement with that expected from the theoretical discussion. This demonstrates the feasibility of using the LSAPS technique to supplement conventional uniaxial stress methods in ambiguous cases.

Finally, we suggest that the demonstrated ability to fully remove the electronic/orientational degeneracy from low-symmetry axial centres in crystalline solids to create a reasonably large number of inequivalent sites in the crystal may have interest for workers in other fields, for example quantum computing.

\section{Acknowledgments}

EM gratefully acknowledges the financial help of the School of Physical Sciences in the purchase of the oriented samples, and in particular the support of Professor E T Kennedy. 


\section{References}

[1] Kaplyanskii A A 1964 Opt. Spektrosk. 16 602-14 (Engl. transl. 1964 Opt. Spectrosc. 16 329-37)

[2] Kaplyanskii A A 1964 Opt. Spektrosk. 16 329-37 (Engl. transl. 1964 Opt. Spectrosc. 16 557-65)

[3] Mohammed K, Davies G and Collins A T 1982 J. Phys. C: Solid State Phys. 15 2779-88

[4] Henry M O, Campion J D, McGuigan K G, Lightowlers E C, Do Carmo M C and Nazare M H 1994 Semicond. Sci. Technol. 9 1375-81

[5] McGuigan K G, Henry M O, Campion J D, Daly S E, McGlynn E and Do Carmo M C 1996 Semicond. Sci. Technol. 11 930-4

[6] O’Morain C, McGuigan K G, Henry M O and Campion J D 1992 Meas. Sci. Technol. 3 337_9

[7] 1995 MATLAB $^{\odot}$ Reference Guide (Natick, MA: MathWorks) p 27

[8] McGlynn E, Henry M O, McGuigan K G and Do Carmo M C 1996 Phys. Rev. B 54 14 494-503

[9] Hughes A E and Runciman W A 1967 Proc. Phys. Soc. 90 827-38

[10] Singh M, Davies G, Lightowlers E C and Watkins G D 1993 Mater. Sci. Forum 117-118 141-6 